\title{
Astroglial wiring is adding complexity to neuroglial networking
}

\author{
Christian Giaume* \\ INSERM U840, Collège de France, Paris, France
}

\section{Edited by:}

Ralf Dringen, University of Bremen,

Germany

\section{Reviewed by:}

Johannes Hirrlinger, Universität

Leipzig, Germany

Joachim Deitmer, University of

Kaisersautern, Germany

*Correspondence:

Christian Giaume, Institut National de la Santé et de la Recherche Médicale

U840, Collège de France, 11 Place

Marcelin Berthelot, 75005 Paris,

France.

e-mail: christian.giaume@college-de-

france.fr
Astrocytes are organized as networks of communicating cells due to their high expression level of connexins, the molecular constituents of gap junction channels. Based on their permeability properties for ions and small signaling molecules such astroglial wiring interferes with neuronal activity and survival. In this paper, I identify and discuss which future technical and conceptual progress or advances should be achieved in order to better understand how neuroglial networking contributes to brain functions and dysfunctions.

Keywords: astrocytes, gap junctions, connexins, glial cells, astroglial networks

\section{INTRODUCTION}

Gap junctions formed by connexins (Cxs), their molecular constituents, are ubiquitous membrane specializations found in all vertebrate tissues. So far 20 and 21 different Cxs have been identified in rodents and humans, respectively (Nagy et al., 2004; Theis et al., 2005). Many of them play critical roles in development and are necessary for tissue functions since several $\mathrm{Cx}$ knock out mice are not viable (Dobrowolski and Willecke, 2009). In the brain, 11 Cxs have been detected (Theis et al., 2005) confirming that Cxs are widely expressed in the central nervous system where they may play important roles. In neurons Cxs provide the morphological basis for electrical synapses which are frequent in the developing brain and also remain in several structures in adults when chemical synapses prevail (Roerig and Feller, 2000; Sohl et al., 2005). However, in the CNS the highest level of Cxs expression occurs in glia, in particular astrocytes, where Cxs are detected from embryonic to adult stages (Dermietzel et al., 1989; Nagy and Rash, 2000).

Gap junctions, at the level of which aggregate intercellular channels, represent a unique example of a direct cytoplasm-tocytoplasm communication. A gap junction channel (GJC) is made of two facing hexamers of $\mathrm{Cxs}$, each of them inserted in the plasma membrane of neighboring cells (Bruzzone et al., 1996). These channels are poorly selective for ions and for small molecular weight signaling molecules $(<1-1.2 \mathrm{kDa})$ (Harris, 2007), providing an ionic and a biochemical or metabolic coupling, respectively. In defined conditions (free divalent solution, metabolic inhibition, inflammation), Cxs in astrocytes can also operate as hemichannels (HCs) defining an inside-outside signaling pathway (see Spray et al., 2006). Finally, pannexins (Pxs), which constitute another family of membrane proteins homologous to the GJC forming proteins in invertebrates called innexins, were shown to form HCs but not GJCs in vertebrates. The expression of functional $\mathrm{Cx}$ and/or Px HCs in astrocytes is still a matter of debate (see Scemes et al., 2007;
Orellana et al., 2009). Although astroglial Cxs are also involved in non-communicating functions (Elias et al., 2007; Scemes, 2008; Cina et al., 2009), here I will limit my comments to glial wiring through networks of communicating astrocytes and thus only consider the GJC function of astroglial Cxs.

In astrocytes, two major Cxs, $\mathrm{Cx} 43$, and $\mathrm{Cx} 30$, are widely expressed allowing intercellular communication for passive dyes between hundreds of astrocytes in certain brain area such as the visual and somatosensory cortex (Binmoller and Muller, 1992; Houades et al., 2006, 2008), the hippocampus (D'Ambrosio et al., 1998; Blomstrand et al., 2004) and the inferior colliculus (Cruz et al., 2007). Although initially considered as a glial syncytium (Mugnaini, 1986; Theis et al., 2005), there is now increasing evidence stating that astrocytes are organized in a more complex and restricted manner forming networks of communicating cells with rules governing their functional status and plasticity (Giaume et al., 2010). Indeed, this astroglial wiring is under the control of neurotransmitters, cytokines, endogenous lipids, and peptides released by neurons and also other brain cell types including microglia and endothelial cells. These multiple regulatory pathways contribute to control the shape and the size of Cx-mediated astroglial networks. Furthermore, in certain brain structures characterized by strong anatomo-functional compartments, astroglial networks overlap with functional units of neurons such as in the somatosensory cortex (Houades et al., 2008) or the olfactory bulb (Roux and Giaume, 2009). In other brain structures the pattern of expression of astroglial Cxs dictates the shaping of these networks such as in the cortex or the hippocampus (Houades et al., 2006). In addition, not all astrocytes located in a coupling domain are connected (Houades et al., 2006, 2008; Rela et al., 2009). Interestingly, this glial wiring allows reconciling two antagonistic concepts of brain organization that opposed the "reticularists" with the defenders of the "Neuron doctrine" more than one century ago. Indeed, while Golgi proposed that neurons 
had fused axons, Cajal argued and finally demonstrated that neurons were individual entities. The widespread GJC-mediated communication in astrocytes demonstrated that astroglial wiring provides a direct cell-to-cell communication in a major cell population of the brain. Now that the concept of dynamic interactions between astrocytes and neurons has gained interest, it is time to consider that their respective modes of communication co-exist and that their interplay has to be taken into account for fully understanding brain functions and dysfunctions.

\section{WHY WE NEED TO KNOW MORE ABOUT ASTROGLIAL NETWORKS}

Neurons control Cx expression in astrocytes and thus regulate astroglial networks (Rouach et al., 2000, 2002; Koulakoff et al., 2008). Reversibly, astroglial Cxs interfere with neuronal differentiation (Wiencken-Barger et al., 2007), migration (Elias et al., 2007), proliferation (Tabernero et al., 2006), and survival (Froger et al., 2010). So it is important to dissect out the molecular and cellular elements sustaining this bidirectional interplay in order to understand how information is processed. However, while synaptic properties and neuronal circuits are well documented, much less is known about astroglial networks. In addition, while multiple findings have strengthened the concept that a loop of dynamic interactions occurs between neurons and astrocytes during the last decade (Haydon and Carmignoto, 2006), most of the studies on neuroglial interactions have been considered solely at the single cell level. Neurotransmitters can be sensed by astrocytes and evoke responses, in particular calcium signals. Interestingly, astrocytes are not excitable cells, but their elaborated calcium signaling confers them a certain mode of "excitability" (Verkhratsky et al., 2002). Finally, there is evidence for calcium-dependent release of "gliotransmitters" (active molecules released by astrocytes that modify neuronal activity) mediated by multiple membrane mechanisms (Volterra, 2002; Malarkey and Parpura, 2008; Agulhon et al., 2010) (but see Agulhon et al., 2010). These three properties (sensing, communicating, and releasing) define a loop of dynamic interactions between neurons and astrocytes (Figure 1A). In addition, the propagation of intercellular calcium waves constitutes a longrange pathway that allows signals to be transmitted to neighboring astrocytes (Cornell-Bell et al., 1990). Cx channels were shown to play a role in this propagating process (see Scemes and Giaume, 2006), which has recently been observed in vivo in normal as well as in a pathological situation (Kuchibhotla et al., 2009). Their occurrence suggests that the above-mentioned loop can involve a larger number of actors (Figure 1B). Astrocytes take up and release neuroactive substances. In addition, if the size of these substances is low enough to permeate GJC, they are expected to be exchanged through GJCs. These statements mean that dynamic neuroglial interactions have also to be considered at a network level. Thus, knowledge about the spatial organization and plasticity of astroglial networks is essential to fully understand neuroglial interactions by considering a more integrated level than the tripartite synapse, which only involves the pre- and postsynaptic elements plus astroglial contacting processes (Figure 2).

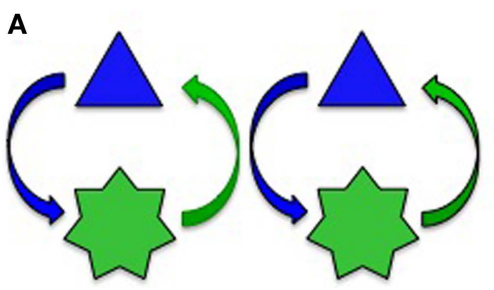

FIGURE 1 | Gap junction-mediated communication enlarges the loop of interaction between neurons (blue triangles) and astrocytes (green stars). Two situations are considered: a one-by-one relationship (A) and a networking that includes the propagation of intercellular signals between adjacent

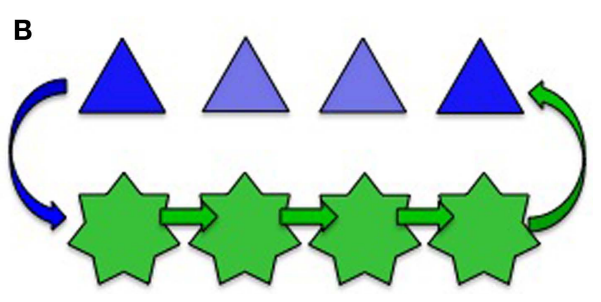

astrocytes (B). This later could occur thanks to calcium waves that involve GJCs, although other alternative pathways exist (see Scemes and Giaume, 2006). As a result, a signal triggered in the neuron on the left can affect the activity of a distant neuron on the right.
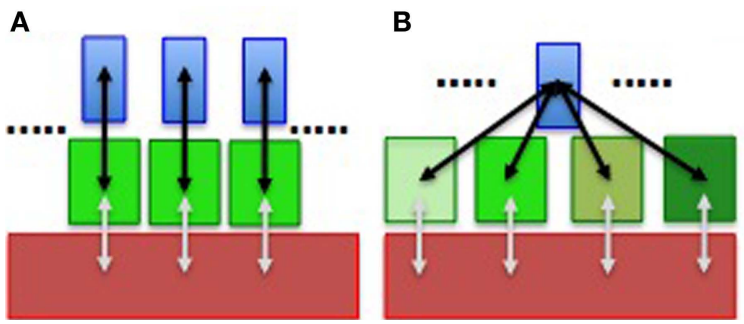

FIGURE 2 | Diagram representing how the wiring of astrocytes adds more complexity to neuroglial networking. Several situations are considered: from a single one-by-one relationship (A), to a multiple relation with a single neuron that contacts several astrocytes (B) and the reverse situation where several neurons make synapses within an astrocytic domain (C). When considering the gap junction-mediated networking of astrocytes, the number of interactions is
C

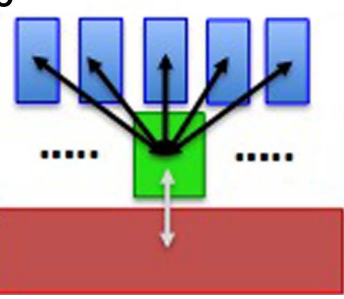

D

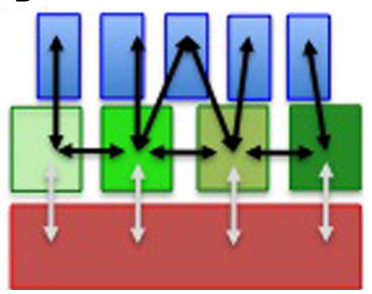

largely increased (D). Note that in (D), the interactions illustrated in (B) and those occurring between neurons themselves are not represented. Finally, the interaction between astrocytes and the vascular system are just suggested (gray arrows). The complex interactions illustrated in (D) (black arrows) are expected to affect the well-established role of astrocytic end-feet in the control of blood flow. 


\section{WHICH NEW TOOLS AND APPROACHES ARE NEEDED?}

To achieve the above goals, several technological and experimental improvements have to be achieved. This means more specific pharmacological agents of $\mathrm{Cx}$ channels, a targeted genetic approach, the development of probes allowing to follow the diffusion and fate of signaling molecules exchanged through astroglial GJCs, a realtime monitoring of their diffusion, and experimental approaches combining in vitro and in vivo models.

\section{NEW PHARMACOLOGICAL AND GENETIC TOOLS}

One major requirement is to selectively block $\mathrm{Cx}$ channels or selectively manipulate the functions of Cx channels. This can be achieved by pharmacological or genetic approaches. However, so far both of them have shown their limits concerning Cx and Pxs channels in glia (see Giaume and Theis, 2010). Indeed, because 11 Cxs and 2 Pxs are expressed in glia and in neurons, several conditions have to be fulfilled to make correct interpretations: (i) the selected approach must have no side effect on the development, the excitability, and the survival of neurons; (ii) as more than one $\mathrm{Cx}$ is expressed, all of them have to be targeted to fully inhibit Cx channels in a defined cell type; (iii) as Cxs support two channel functions, it is important to be able to discriminate between GJC and HC activities; and finally, (iv) as Pxs can form HCs, their contribution should be distinguished. Up to now, glycyrrhetinic acid derivatives, including carbenoxolone, are the most used uncoupling agents, but they do not discriminate between $\mathrm{Cx}$ and Px channels (Bruzzone et al., 2005) and they have side effects on neurons (Juszczak and Swiergiel, 2009). Similarly, while Cx gene ablation can be specific to individual Cxs and to glial cell types, this approach often lacks sufficient temporal control. In addition, so far there are at least two major limitations of $\mathrm{Cx} 43$ gene ablation strategy applied to glial Cxs: (i) changes in gene expression were observed in $\mathrm{Cx} 43 \mathrm{KO}$ mice (Iacobas et al., 2007) limiting the interpretation of the observed phenotypical changes; (ii) when $\mathrm{Cx} 43$ is missing in astrocytes, it is difficult to define its major mechanism of action under normal conditions (intercellular coupling, HC activity, adhesion or control of gene expression). In the future, several aims have to be challenged: (i) for a pharmacological approach, the objective will be to design molecules that can specifically block GJCs or HCs. This goal will be rather difficult to achieve for GJCs as both of their extremities are cytoplasmic which limits their access. In contrast, HC blockers should be easier to develop as one end of the channel faces the extracellular space; (ii) for a genetic approach, the objective will be to elucidate the mechanisms by which Cxs exert their different functions (GJCs and HCs, adhesion or control of gene expression). This goal will be achieved by using a combination of engineered mice. As for instance, the Cx43G138R mouse that exhibits a lost in GJC but preserves HC function (Dobrowolski et al., 2008), and again (iii) it will be important to distinguish between $\mathrm{Cx}$ versus Px contribution to HCs. An alternative way will be to use RNA interference (RNAi) to down-regulate gene expression using small RNAs with designed target mRNA complementary sequences. This approach has been successfully used to study the role of Cxs in neurogenesis (Elias et al., 2007; Cina et al., 2009), in the control of astroglial gene expression (Figiel et al., 2007; Iacobas et al., 2008; Olk et al., 2009), as well as in astrocyte proliferation and glucose transport (Herrero-Gonzalez et al., 2009). Clear advantages of the RNAi approach are its flexibility, short preparation time, and temporal control compared to gene ablation. However, it is limited by its local action and its lower efficacy leading to knockdown rather than knockout. Also, the development of a lentiviral strategy to specifically enhance or silence the expression of astroglial Cxs starts to be available. Indeed, recently engineered lentiviral constructs that specifically target astrocytes have been generated (Colin et al., 2009). As it is also the case for RNAi, the transgene expression is restricted to the injected brain region and the contralateral side can be taken as an internal control. Finally, retroviral delivery of cre recombinase has recently been used in the hippocampus to study the impact of gene function in dividing cell populations by performing injections of a Cre-expressing retrovirus into Cx30-/-/ Cx43fl/fl mice (Kunze et al., 2009).

\section{DEVELOPMENT OF ON-LINE MONITORING OF BIOCHEMICAL COUPLING}

New approaches and more appropriate tools are needed to assess the strength, the spatial organization, and the dynamic of gap junction-mediated communication in astrocytes. While the double patch-clamp technique can be fully used with cultured astrocytes to obtain quantitative data of GJCs, even at the single channel level (Giaume et al., 1991), its application in brain slices and in vivo is limited. This is mainly due to the low input resistance and the short length constant of astroglial membrane and to the connection of more than two astrocytes in the $3 \mathrm{D}$ in vivo situation. Although the kinetics of coupling changes can be followed, these features limit the accuracy of quantitative measurements of junctional currents (Meme et al., 2009).

Alternatively, for more that 20 years gap junctional communication in astroglial cells has been extensively studied using dye coupling techniques mostly in vitro. Using passive dyes this technique allows determining the level of coupling at a define moment within a population of astrocytes and provides a map of their communication pathways. However, this technique does not inform about the kinetics of GJCs and the nature of signaling molecules exchanged between cells. Accordingly, new technological developments are required to follow biochemical coupling between astrocytes and to understand the contribution of astroglial networks to neuroglial interactions. This could be achieved by setting time-lapse monitoring of dye diffusion in brain slices or in vivo using patch-clamp recording, fluorescent dyes, and two-photon microscopy.

The identification of the biochemical dialog through astroglial networks could be achieved by the development of new tagged signaling molecules and the application of methods to follow online changes of second messenger concentration (see for instance Vincent et al., 2008) within a population of coupled astrocytes. Recently, such improvement has started to be carried out for energy metabolites by using fluorescent glucose derivatives in hippocampal slices to visualize astroglial metabolic networks and demonstrating their activity-dependent regulation by neurons (Rouach et al., 2008).

\section{FROM CULTURES TO IN VIVO MODELS}

Obviously the study of astroglial networks will have to follow the general movement that in neurosciences has led many laboratories to set in vivo models. In this domain, the development of optic and imaging techniques will contribute to demonstrate the occurrence 
of astroglial networks and to observe dynamic changes in their size and shape. The possibility to follow the intercellular exchange of signaling molecules will likely show us that the extent of biochemical coupling is more specific and restricted than anticipated. This is due to the fact that signaling molecules have a determined radius of action and a life-time that limit their domain of diffusion unless a regenerative process is involved. Also, natural triggering signals that initiate an intercellular propagation are expected to be weaker in amplitude and shorter in duration compared to the high concentration and prolonged application reached when dye injections are performed. However, some regenerative or semi-regenerative processes could contribute to amplify the propagation of these signaling molecules as already proposed for intercellular calcium waves and IP3 signaling (Giaume and Venance, 1998; Hofer et al., 2002). When such in vivo approaches to monitor astroglial networks will be combined with imaging of neuronal circuit activity, it will be possible to study neuroglial interactions at the networking level. In these conditions, the silencing of astroglial communication will allow investigating the role of GJCs. Such in vivo approach does not exclude the use of other models, since cultures and co-cultures provide a unique way to study the contribution of each cell type separately and acute slices allow studying deep brain areas difficult to access in vivo and is more appropriate for pharmacological manipulations. Of course the more informative situation will be offered by combining these three types of preparation. For in vivo studies, several sensory systems seem to be appropriate because they offer the possibility to work with natural stimuli. For instance, the somatosensory cortex (see Giaume et al., 2009) is an interesting model because one functional unit corresponding to a "barrel" can be activated by the stimulation of a single whisker while in vivo on-line imaging of population of astrocytes can be achieved (Wang et al., 2006). Interestingly, it was recently reported that GJCmediated communication is favored within a barrel and oriented toward its center (Houades et al., 2008). This restricted astroglial wiring within each barrel parallels the organization of the excitatory neuronal circuits and favors neuroglial networking within a single functional unit.

\section{WHAT ARE WE LOOKING FOR AND WHICH FUNCTIONS OF ASTROGLIAL NETWORKS SHOULD BE STUDY? IONIC HOMEOSTASIS}

As GJC are permeable to ions with a poor selectivity (Harris, 2007), they should certainly be important for ionic homeostasis. In fact, this was their first proposed function when glial cells were initially characterized as being highly permeable to $\mathrm{K}^{+}$and strongly coupled. This led to propose that glial gap junctions could drive a "spatial buffer" current, which could move $\mathrm{K}^{+}$from active sites to a distal region (Orkand et al., 1966). Since then, a better understanding of this phenomenon has been achieved and led to the conclusion that, at least in the hippocampus, gap junction-dependent processes in astrocytes only partially account for $\mathrm{K}^{+}$buffering (Wallraff et al., 2006). Recent double patch-clamp studies permitted to monitor junctional currents between pairs of astrocytes recorded in acute hippocampal slices (Meme et al., 2009; Xu et al., 2010) and revealed that the averaged coupling ratio is very low $(<5 \%)$. Moreover, only about $2 \%$ of locally elevated $\mathrm{K}^{+}$, assuming to occur at the soma, can travel across GJCs between a pair of astrocytes (Xu et al., 2010). Also, because in the hippocampus one astrocyte contacts about a dozen of other astrocytes in the 3D situation, $20-25 \%$ of $\mathrm{K}^{+}$-induced currents can travel from any astrocytic soma to the GJCs of the nearest neighboring astrocytes. Because during neuronal activity the changes in the extracellular $\mathrm{K}^{+}$concentration are small $(>1 \mathrm{mM})$, it is expected that only few $\mathrm{K}^{+}$ions will travel further away from the nearest neighboring astrocyte. These observations have led to state that $\mathrm{K}^{+}$currents cannot go further than the astrocytes that are directly connected to the astrocyte exhibiting the initial rise in $\mathrm{K}^{+}$(Xu et al., 2010). Alternatively, the elaborated calcium signaling in astrocytes and the permeability of GJCs for $\mathrm{Ca}^{2+}$ and IP3 may provide a better example for the contribution of GJCs to astroglial physiology. Indeed, while membrane excitability is required for action potential propagation in neurons, calcium signaling in astrocytes seems to be an alternative mode of intercellular propagation of information. It is now well established that astrocytes display spontaneous and evoked intracellular calcium concentration $\left(\left[\mathrm{Ca}^{2+}\right]_{\mathrm{i}}\right)$ increases that provide the basis for a form of astrocyte excitability (Fiacco and McCarthy, 2006). Moreover, the observation of calcium waves between astrocytes has provided arguments to state that increases in $\left[\mathrm{Ca}^{2+}\right]_{i}$ can also propagate between astrocytes (see paragraph 2) a process to which HCs and/or GJCs (in particular Cx43) are thought to participate (Fiacco and McCarthy, 2006; Scemes and Giaume, 2006). However, their occurrence is still matter of debate (Agulhon et al., 2008; Barres, 2008). Consequently, to better understand the role of GJCs in the physiology of astrocytes and their interaction with neurons several questions remain to be answered concerning their contribution to ionic homeostasis and intercellular signaling. In the future, these points should be addressed using new models and technical approaches (see paragraph 3 ).

\section{ASTROGLIAL WIRING AND BIOCHEMICAL COUPLING}

Thanks to their permeability to energy substrates (glucose, lactate...) GJC contributes to the role played by astrocytes in brain metabolism. Indeed, astrocytes occupy a strategic location between the vasculature, the source of energy compounds, and the neurons, the main energy consumer. In addition, perivascular astrocytes send end-feet that enwrap blood vessels and capillaries (Mathiisen et al., 2010; Iadecola and Nedergaard, 2007). Consequently, their wiring properties provide intercellular pathways contributing to establish a metabolic homeostasis in the brain through the balance between the delivery and the dissipation of energy compounds. The diffusion of energy substrates through such metabolic networks was recently studied in hippocampal slices using fluorescent glucose derivatives (Rouach et al., 2008) and a real-time enzymatic fluorescent assay lactate in inferior colliculus slices (Gandhi et al., 2009). In the near future, these pioneering works should be extended using in vivo preparations as soon as adequate fluorescent probes will allow following glucose and its derivatives once in the brain.

In addition to $\mathrm{Ca}^{2+} / \mathrm{IP}_{3}$ (Venance et al., 1997; Kang et al., 2005) and energy metabolites (Tabernero et al., 1996; Blomstrand and Giaume, 2006; Ball et al., 2007; Rouach et al., 2008; Gandhi et al., 2009), astrocytes GJCs are also permeable to amino acids (see Giaume et al., 1997), glutathione, and ATP/ADP/AMP (Goldberg et al., 1999, 2002). In the future, the role of these potential exchanges of signaling molecules will have to be examined. Finally, the possibility of exchange of neuroprotective and neurotoxic molecules between astrocytes should be also taken 
into account when investigating their role in neuroinflammation. Indeed, there is already evidence that neuronal survival is affected when astroglial Cxs are lacking in several pathological models (see Giaume et al., 2007, 2010). However, the literature is rather confusing about their beneficial or deleterious role. This is likely due to the difficulty to discriminate between the contribution of astroglial HCs and GJCs. To answer this question more specific tools are required (see paragraph 3 ).

\section{PHARMACOLOGICAL HETEROGENEITY OF ASTROCYTES}

There is increasing evidence for morphological and functional heterogeneity of astrocytes, including the expression of membrane receptors for sensing neurotransmitters (see Matyash and Kettenmann, 2010). This heterogeneity is observed when comparing different brain areas but is also true within a defined structure, suggesting that neighboring astrocytes differentially integrate neuronal activity. Considering that the stimulation of metabotropic receptors generates changes in second messenger concentration and that GJCs are permeable to most of them, the existence of a high level of gap junctionmediated communication is expected to mask, or at least attenuate, the consequences of a pharmacological heterogeneity in a group of coupled astrocytes. Such question was already addressed using primary cultures of astrocytes and analyzing their pattern of calcium responses to agonist stimulation of several membrane receptors expressed either in all or only in a subset of astrocytes (Venance et al., 1998). When the occurrence of agonist-induced calcium responses is compared in a situation with open or closed GJCs, a difference in the number of responding cells is observed for heterogeneously distributed receptors. Interestingly, a similar observation has been reported when monitoring the level of intracellular sodium concentration in cultured astrocytes (Rose and Ransom, 1997). The next step will be to study such role in vivo and to determine whether the level of gap junctional communication affects the occurrence and the shape of receptor-mediated responses in astrocytes.

\section{REFLEXIVE GAP JUNCTIONS AND SUBCELLULAR MICRODOMAINS}

One important discovery in the glial field is the demonstration that astrocytes occupy individual domains that do not overlap (Bushong et al., 2002; Ogata and Kosaka, 2002). At first sight, this observation should minimize the role of intercellular GJCs since gap junctions are located at this limited contact area between two neighboring astrocytes. However, the demonstration of a metabolic rescue of synaptic transmission in hypoglycemic condition demonstrates that this restricted zone of contact is sufficient to provide a functional role of intercellular GJCs by establishing a metabolic coupling within an astroglial network (Rouach et al., 2008). Also, the "calcium-clamp" achieved by infusing astroglial networks with the calcium chelator BAPTA results in the silencing of coupled hippocampal astroytes

\section{REFERENCES}

Agulhon, C., Fiacco, T. A., and McCarthy, K.D. (2010). Hippocampal short- and long-term plasticity are not modulated by astrocyte $\mathrm{Ca}^{2+}$ signaling. Science 327, 1250-1254.

Agulhon, C., Petravicz, J., McMullen, A. B., Sweger, E. J., Minton, S. K., Taves, S. R., Casper, K. B., Fiacco,

(Serrano et al., 2006; Henneberger et al., 2010) demonstrating that GJC-mediated communication is sufficiently high to be used to modify a signaling property of astrocytes.

In addition to cell-to-cell communication, Cxs can also operate as GJCs between processes originating from a single astrocyte. Such "reflexive" gap junctions, already described at the ultrastructural level (Rohlmann and Wolff, 1998) could contribute to astrocytic subcellular microdomains (Grosche et al., 1999) and to their integrative responses to neuronal inputs. As a single astrocyte contacts a large number of synapses it is expected that the existence of such microdomains are relevant for brain function. Unfortunately, the numerous gap junctions that astrocytes establish with their neighbors limit the study of the properties and role of these reflexive GJC. One possibility will be to work with freshly isolated astrocytes that are dissociated from the others (Reichenbach et al., 2010), but maintain their morphology and hopefully their reflexive GJCs. Thus by using this method, electrophysiological properties and calcium responses in astrocytes could be compare when GJCs are inhibited either by pharmacological or genetic approaches. This will allow investigating the role of reflexive GJCs in the physiology of a single astrocyte.

\section{CONCLUSIONS}

Dynamic neuroglial interaction is a property that starts to be taken into consideration to understand how neuronal activity and synaptic transmission are regulated (Haydon and Carmignoto, 2006; Agulhon et al., 2008; Barres, 2008). But so far, the strong level of intercellular communication occurring between groups of astrocytes has been neglected. In the future, considering this more integrated level of neuroglial networking will certainly help better understanding how brain circuits work. Here, I have listed some of the technological improvements and conceptual progresses that are required to really make a significant breakthrough in this direction. Brain pathologies and the role of glia in these processes are certainly other situations in which astroglial networking may play a role (Giaume et al., 2007). Indeed, there are numerous report of changes in the expression of astroglial Cxs in neurodegenerative diseases, ischemia, epilepsy, and brain injuries (see Giaume et al., 2010). No doubt that advances made in the study of Cx-based channels and astroglial networks will contribute in the near future to open new ways to understand brain functions and dysfunctions.

\section{ACKNOWLEDGMENTS}

I wish to thank Dr N. Rouach and Pr A. Bordey for her critical comments on this article and to express my gratitude to all the collaborators and colleagues who along the years have contributed to improve my understanding of connexins function in the brain. Their work, ideas, and shared discussions constitute the basement of this article.

lus: implications for $[(14) \mathrm{C}]$ glucose metabolite trafficking. J. Neurosci. Res. $85,3267-3283$.

Barres, B. A. (2008). The mystery and magic of glia: a perspective on their roles in health and disease. Neuron $60,430-440$.

Binmoller, F. J., and Muller, C. M. (1992). Postnatal development of dye-coupling among astrocytes in rat visual cortex. Glia 6, 127-137.

Blomstrand, F., and Giaume, C. (2006) Kinetics of endothelin-induced inhibition and glucose permeability of astrocyte gap junctions. J. Neurosci. Res. 83, 996-1003.

Astrocytic con and rapid, extensive dye transfer via gap junctions in the inferior collicu-
Blomstrand, F., Venance, L., Siren, A. L., Ezan, P., Hanse, E., Glowinski, 
J., Ehrenreich, H., and Giaume, C. (2004). Endothelins regulate astrocyte gap junctions in rat hippocampal slices. Eur. J. Neurosci. 19, 1005-1015.

Bruzzone, R., Barbe, M. T., Jakob, N. J., and Monyer, H. (2005). Pharmacological properties of homomeric and heteromeric pannexin hemichannels expressed in Xenopus oocytes. J. Neurochem. 92, 1033-1043.

Bruzzone, R., White, T.W., and Paul, D. L. (1996). Connections with connexins: the molecular basis of direct intercellular signaling. Eur. J. Biochem. 238, 1-27.

Bushong, E. A., Martone, M. E., Jones, Y. Z., and Ellisman, M. H. (2002). Protoplasmic astrocytes in CAl stratum radiatum occupy separate anatomical domains. J. Neurosci. 22, 183-192.

Cina, C., Maass, K., Theis, M., Willecke, K., Bechberger, J. F., and Naus, C. C. (2009). Involvement of the cytoplasmic C-terminal domain of connexin 43 in neuronal migration. J. Neurosci. 29, 2009-2021.

Colin, A., Faideau, M., Dufour, N., Auregan, G., Hassig, R., Andrieu, T., Brouillet, E., Hantraye, P., Bonvento, G., and Deglon, N. (2009). Engineered lentiviral vector targeting astrocytes in vivo. Glia 57, 667-679.

Cornell-Bell, A. H., Finkbeiner, S. M. Cooper, M. S., and Smith, S. J. (1990). Glutamate induces calcium waves in cultured astrocytes: long-range glial signaling. Science 247, 470-473.

Cruz, N. F., Ball, K. K., and Dienel, G. A. (2007). Functional imaging of focal brain activation in conscious rats: impact of [(14)C]glucose metabolite spreading and release. J. Neurosci. Res. $85,3254-3266$.

D’Ambrosio, R., Wenzel, J., Schwartzkroin, P. A., McKhann, G. M. 2nd, and Janigro, D. (1998). Functional specialization and topographic segregation of hippocampal astrocytes. J. Neurosci. $18,4425-4438$.

Dermietzel, R., Traub, O., Hwang, T. K., Beyer, E., Bennett, M. V., Spray, D. C., and Willecke, K. (1989). Differential expression of three gap junction proteins in developing and mature brain tissues. Proc. Natl. Acad. Sci. U.S.A. 86, 10148-10152.

Dobrowolski, R., Sasse, P., Schrickel, J. W., Watkins, M., Kim, J. S., Rackauskas, M., Troatz, C., Ghanem, A., Tiemann, K., Degen, J., Bukauskas, F. F., Civitelli, R., Lewalter, T., Fleischmann, B. K., and Willecke, K. (2008). The conditional connexin43G138R mouse mutant represents a new model of hereditary oculodentodigital dysplasia in humans. Hum. Mol. Genet. 17, 539-554.
Dobrowolski, R., and Willecke, K. (2009). Connexin-caused genetic diseases and corresponding mouse models. Antioxid. Redox Signal. 11, 283-295.

Elias, L. A., Wang, D. D., and Kriegstein, A. R. (2007). Gap junction adhesion is necessary for radial migration in the neocortex. Nature 448, 901-907.

Fiacco, T. A., and McCarthy, K. D. (2006). Astrocyte calcium elevations: properties, propagation, and effects on brain signaling. Glia 54, 676-690.

Figiel, M., Allritz, C., Lehmann, C., and Engele, J. (2007). Gap junctional control of glial glutamate transporter expression. Mol. Cell. Neurosci. 35, 130-137.

Froger, N., Orenalla, J. A., Calvo, C. F., Amigou, E., Kozoriz, M. G., Naus, C. C., Sáez, J. C., and Giaume, C. (2010). Inhibition of cytokine-induced connexin43 hemichannel activity in astrocytes is neuroprotective. $\mathrm{Mol}$. Cell. Neurosci. 45, 37-46.

Gandhi, G. K., Cruz, N. F., Ball, K. K., Theus, S. A., and Dienel, G. A. (2009) Selective astrocytic gap junctional trafficking of molecules involved in the glycolytic pathway: impact on cellular brain imaging. J. Neurochem. 110, 857-869.

Giaume, C., Fromaget, C., el Aoumari, A., Cordier, J., Glowinski, J., and Gros, D. (1991). Gap junctions in cultured astrocytes: single-channel currents and characterization of channel-forming protein. Neuron 6, 133-143.

Giaume, C., Kirchhoff, F., Matute, C. Reichenbach, A., and Verkhratsky, A. (2007). Glia: the fulcrum of brain diseases. Cell Death Differ. 14, 1324-1335.

Giaume, C., Koulakoff, A., Roux, L., Holcman, D., and Rouach, N. (2010). Astroglial networks: a step further in neuroglial and gliovascular interactions. Nat. Rev. Neurosci. 11, 87-99.

Giaume, C., Maravall, M., Welker, E., and Bonvento, G. (2009). The barrel cortex as a model to study dynamic neuroglial interaction. Neuroscientist. 15, 351-366

Giaume, C., Tabernero, A., and Medina, J. M. (1997). Metabolic trafficking through astrocytic gap junctions. Glia 21, 114-123.

Giaume, C., and Theis, M. (2010) Pharmacological and genetic approaches to study connexin-mediated channels in glial cells of the central nervous system. Brain Res. Rev. 63, 160-176.

Giaume, C., and Venance, L. (1998). Intercellular calcium signaling and gap junctional communication in astrocytes. Glia 24, 50-64.
Goldberg, G. S., Lampe, P. D., and Nicholson, B. J. (1999). Selective transfer of endogenous metabolites through gap junctions composed of different connexins. Nat. Cell Biol. 1, 457-459.

Goldberg, G. S., Moreno, A. P., and Lampe, P. D. (2002). Gap junctions between cells expressing connexin 43 or 32 show inverse permselectivity to adenosine and ATP. J. Biol. Chem. 277, 36725-36730.

Grosche, J., Matyash, V., Moller, T., Verkhratsky, A., Reichenbach, A., and Kettenmann,H. (1999). Microdomains for neuron-glia interaction: parallel fiber signaling to Bergmann glial cells. Nat. Neurosci. 2, 139-143.

Harris, A. L. (2007). Connexin channel permeability to cytoplasmic molecules. Prog. Biophys. Mol. Biol. 94 120-143.

Haydon, P. G., and Carmignoto, G. (2006) Astrocyte control of synaptic transmission and neurovascular coupling. Physiol. Rev. 86, 1009-1031.

Henneberger, C., Papouin, T., Oliet, S H., and Rusakov, D. A. (2010). Longterm potentiation depends on release of D-serine from astrocytes. Nature 463, 232-236.

Herrero-Gonzalez, S., Valle-Casuso, J. C., Sanchez-Alvarez, R., Giaume, C., Medina, J. M., and Tabernero, A (2009). Connexin 43 is involved in the effect of endothelin-1 on astrocyte proliferation and glucose uptake. Glia 57, 222-233.

Hofer, T., Venance, L., and Giaume, C. (2002). Control and plasticity of intercellular calcium waves in astrocytes: a modeling approach. J. Neurosci. 22 4850-4859.

Houades, V., Koulakoff,A., Ezan, P., Seif, I. and Giaume, C. (2008). Gap junctionmediated astrocytic networks in the mouse barrel cortex. J. Neurosci. 28 5207-5217.

Houades, V., Rouach, N., Ezan, P., Kirchhoff, F., Koulakoff, A., and Giaume, C. (2006). Shapes of astrocyte networks in the juvenile brain. Neuron Glia Biol. 2, 3-14.

Iacobas, D. A., Iacobas, S., and Spray, D. C. (2007). Connexin43 and the brain transcriptome of newborn mice. Genomics 89, 113-123.

Iacobas, D. A., Iacobas, S., UrbanMaldonado, M., Scemes, E., and Spray, D. C. (2008). Similar transcriptomic alterations in $\mathrm{Cx} 43$ knockdown and knockout astrocytes. Cell Commun. Adhes. 15, 195-206.

Iadecola, C., and Nedergaard, M. (2007). Glial regulation of the cerebral microvasculature. Nat. Neurosci. 10 1369-1376.

Juszczak, G. R., and Swiergiel, A. H. (2009). Properties of gap junction blockers and their behavioural, cognitive and electrophysiological effects: animal and human studies. Prog. Neuropsychopharmacol. Biol. Psychiatry 33, 181-198.

Kang, N., Xu, J., Xu, Q., Nedergaard, M., and Kang, J. (2005). Astrocytic glutamate release-induced transient depolarization and epileptiform discharges in hippocampal CA1 pyramidal neurons. J. Neurophysiol. 94, 4121-4130.

Koulakoff, A., Ezan, P., and Giaume, C. (2008). Neurons control the expression of connexin 30 and connexin 43 in mouse cortical astrocytes. Glia 56, 1299-1311.

Kuchibhotla, K. V., Lattarulo, C. R. Hyman, B. T., and Bacskai, B. J. (2009). Synchronous hyperactivity and intercellular calcium waves in astrocytes in Alzheimer mice. Science $323,1211-1215$.

Kunze, A., Congreso, M. R., Hartmann, C., Wallraff-Beck, A., Huttmann, K., Bedner, P., Requardt, R., Seifert, G. Redecker, C., Willecke, K., Hofmann, A., Pfeifer, A., Theis, M., and Steinhauser, C. (2009). Connexin expression by radial glia-like cells is required for neurogenesis in the adult dentate gyrus. Proc. Natl. Acad. Sci. U.S.A. 106, 11336-11341.

Malarkey, E. B., and Parpura, V. (2008). Mechanisms of glutamate release from astrocytes. Neurochem. Int. 52 , 142-154.

Mathiisen, T. M., Lehre, K. P., Danbolt, N. C., Ottersen, O.P. (2010). The perivascular astroglial sheath provides a complete covering of the brain microvessels: an electron microscopic 3D reconstruction. Glia 58, 1094-1103.

Matyash, V., and Kettenmann, H. (2010). Heterogeneity in astrocyte morphology and physiology. Brain Res. Rev. 63, 2-10.

Meme, W., Vandecasteele, M., Giaume, C., and Venance, L. (2009). Electrical coupling between hippocampal astrocytes in rat brain slices. Neurosci. Res. 63, 236-243.

Mugnaini, E. (1986). "Cell junctions of astrocytes, ependymal and related cells in the mammal central nervous system, with emphasis on the hypothesis of a generalized syncytium of supporting cells," in Astrocytes, eds S. Fedoroff and A. Vernadakis (New York: Academic Press), 329-371.

Nagy, J. I., Dudek, F. E., and Rash, J. E. (2004). Update on connexins and gap junctions in neurons and glia in the mammalian nervous system. Brain Res. Brain Res. Rev. 47, 191-215.

Nagy, J.I., and Rash, J.E. (2000).Connexins and gap junctions of astrocytes and oligodendrocytes in the CNS. Brain Res. Brain Res. Rev. 32, 29-44. 
Ogata, K., and Kosaka, T. (2002). Structural and quantitative analysis of astrocytes in the mouse hippocampus. Neuroscience 113, 221-233.

Olk, S., Zoidl, G., and Dermietzel, R. (2009). Connexins, cell motility, and the cytoskeleton. Cell Motil. Cytoskeleton 66, 1000-1016.

Orellana, J. A., Saez, P. J., Shoji, K. F., Schalper, K. A., Palacios-Prado, N., Velarde, V., Giaume, C., Bennett, M. V., and Saez, J. C. (2009). Modulation of brain hemichannels and gap junction channels by pro-inflammatory agents and their possible role in neurodegeneration. Antioxid. Redox Signal. 11, 369-399.

Orkand, R. K., Nicholls, J. G., and Kuffler, S. W. (1966). Effect of nerve impulses on the membrane potential of glial cells in the central nervous system of amphibia. J. Neurophysiol. 29, 788-806.

Reichenbach, A., Derouiche, A., and Kirchhoff, F. (2010). Morphology and dynamics of perisynaptic glia. Brain Res. Rev. 63, 11-25.

Rela, L., Bordey, A., and Greer, C. A. (2009). Olfactory ensheathing cell membrane properties are shaped by connectivity. Glia 58, 665-678.

Roerig, B., and Feller, M. B. (2000). Neurotransmitters and gap junctions in developing neural circuits. Brain Res. Brain Res. Rev. 32, 86-114.

Rohlmann, A., and Wolff, J. R. (1998). "Subcellular topography and plasticity of gap junction distribution in astrocytes," in Gap Junctions in the Nervous System,eds D.C.Spray and R. Dermietzel (Austin, TX: R. G. Landes), 175-192.

Rose, C. R., and Ransom, B. R. (1997). Gap junctions equalize intracellular $\mathrm{Na}+$ concentration in astrocytes. Glia 20, 299-307.
Rouach, N., Glowinski, J., and Giaume, C. (2000). Activity-dependent neuronal control of gap-junctional communication in astrocytes. J. Cell Biol. 149, 1513-1526.

Rouach, N., Koulakoff, A., Abudara, V., Willecke, K., and Giaume, C. (2008). Astroglial metabolic networks sustain hippocampal synaptic transmission. Science 322, 1551-1555.

Rouach, N., Tence, M., Glowinski, J., and Giaume, C. (2002). Costimulation of $\mathrm{N}$-methyl-D-aspartate and muscarinic neuronal receptors modulates gap junctional communication in striatal astrocytes. Proc. Natl. Acad. Sci. U.S.A. 99, 1023-1028.

Roux, L., and Giaume, C. (2009). Two astroglial neworks are differentially regulated by neuronal activity in the olfactory glomerular layer. Glia 57(Suppl. 13), 57.

Scemes, E. (2008). Modulation of astrocyte P2Y1 receptors by the carboxyl terminal domain of the gap junction protein Cx43. Glia 56, 145-153.

Scemes, E., and Giaume, C. (2006). Astrocyte calcium waves: what they are and what they do. Glia 54, 716-725.

Scemes, E., Suadicani, S. O., Dahl, G., and Spray, D.C. (2007). Connexin and pannexin mediated cell-cell communication. Neuron Glia Biol. 3, 199-208.

Serrano,A., Haddjeri, N.,Lacaille, J.C., and Robitaille, R. (2006). GABAergic network activation of glial cells underlies hippocampal heterosynaptic depression. J. Neurosci. 26, 5370-5382.

Sohl, G., Maxeiner, S., and Willecke, K. (2005). Expression and functions of neuronal gap junctions. Nat. Rev. Neurosci. 6, 191-200.

Spray, D. C., Ye, Z. C., and Ransom, B. R. (2006). Functional connexin "hemichannels": a critical appraisal. Glia 54, 758-773.

Tabernero, A., Giaume, C., and Medina, J. M. (1996).Endothelin-1 regulates glucose utilization in cultured astrocytes by controlling intercellular communication through gap junctions. Glia 16, 187-195.

Tabernero, A., Medina, J.M., and Giaume, C. (2006). Glucose metabolism and proliferation in glia: role of astrocytic gap junctions. J. Neurochem. 99 1049-1061.

Theis, M., Sohl, G., Eiberger, J., and Willecke, K. (2005). Emerging complexities in identity and function of glial connexins. Trends Neurosci. 28 188-195.

Venance, L., Premont, J., Glowinski, J., and Giaume, C. (1998). Gap junctional communication and pharmacological heterogeneity in astrocytes cultured from the rat striatum. J. Physiol. (Lond.) 510, 429-440.

Venance, L., Stella, N., Glowinski, J. and Giaume, C. (1997). Mechanism involved in initiation and propagation of receptor-induced intercellular calcium signaling in cultured rat astrocytes. J. Neurosci. 17, 1981-1992.

Verkhratsky, A., Landfield, P. W., and Thibault, O. (2002). Preface. $\mathrm{Ca}^{2+}$ and neuronal pathology. Eur. J. Pharmacol. 447, 115-117.

Vincent, P., Gervasi, N., and Zhang, J. (2008). Real-time monitoring of cyclic nucleotide signaling in neurons using genetically encoded FRET probes. Brain Cell Biol. 36, 3-17.

Volterra, A., and Bezzi, P. (2002). "Release of transmitters from glial cells," in The Tripartite Synapse: Glial in Synaptic Transmission eds A. Volterra, P. Magistretti, and P.G. Haydon (Oxford: Oxford University Press), 164-182.
Wallraff, A., Kohling, R., Heinemann, U., Theis, M., Willecke, K., and Steinhauser, C. (2006). The impact of astrocytic gap junctional coupling on potassium buffering in the hippocampus. J. Neurosci. 26, 5438-5447.

Wang, X., Lou, N., Xu, Q., Tian, G. F., Peng, W. G., Han, X., Kang, J., Takano, T., and Nedergaard, M. (2006). Astrocytic $\mathrm{Ca} 2+$ signaling evoked by sensory stimulation in vivo. Nat. Neurosci. 9 , 816-823.

Wiencken-Barger,A.E., Djukic, B., Casper, K. B., and McCarthy, K. D. (2007). A role for Connexin43 during neurodevelopment. Glia 55, 675-686.

Xu, G., Wang, W., Kimelberg, H. K., and Zhou, M. (2010). Electrical coupling of astrocytes in rat hippocampal slices under physiological and simulated ischemic conditions. Glia 58, 481-493.

Conflict of Interest Statement: The author declares that the research was conducted in the absence of any commercial or financial relationships that could be construed as a potential conflict of interest.

Received:03 July 2010; paper pending published: 19 July 2010; accepted: 23 August 2010; published online: 20 September 2010.

Citation: Giaume C (2010) Astroglial wiring is adding complexity to neuroglial networking. Front. Neuroenerg. 2:129. doi: 10.3389/fnene.2010.00129

Copyright (c) 2010 Giaume. This is an open-access article subject to an exclusive license agreement between the authors and the Frontiers Research Foundation, which permits unrestricted use, distribution, and reproduction in any medium, provided the original authors and source are credited. 